\title{
Factores socio-ambientales y de conservación en predios amazónicos de Ecuador vinculados o no al programa Socio Bosque
}

\section{Socio-environmental and conservation factors in Amazonian lands of Ecuador linked or not to the Socio Bosque program}

Patricio YÁNEZ M.

Universidad Internacional del Ecuador, Ecuador

Universidad Iberoamericana del Ecuador, Ecuador

María J. GRANDA

Municipio del Distrito Metropolitano de Quito, Ecuador

Autor para correspondencia: apyanez@ hotmail.com

Fecha de recepción: 01 de Septiembre de 2016 - Fecha de aceptación: 01 de Noviembre de 2016

Resumen: el presente estudio explora aspectos relacionados con la conservación de bosques del occidente de la región amazónica ecuatoriana (provincias de Napo, Pastaza y Morona Santiago) y la relación de ésta con algunas variables sociales y económicas. Los datos se generaron en ciento veinte y dos predios de dos grupos de finqueros: unos pertenecientes a la iniciativa Socio Bosque y otros no, la fase de campo se realizó entre febrero a marzo de 2014. En la información recogida, se incluyeron aspectos geográficos, sociales, laborales, económicos, de uso del suelo y de actividades futuras. Entre otros resultados, se evidencia que la mayoría de propietarios desea mantener sus zonas boscosas con especies nativas, pero manifiesta la necesidad de contar con capacitaciones más frecuentes en torno a la dinámica de los ecosistemas locales y las formas más idóneas para cuidarlos.

Palabras claves: región amazónica ecuatoriana; programa socio bosque; aspectos socioeconómicos; conservación de bosques; pérdida de biodiversidad

\begin{abstract}
This study explores issues related to the conservation of forests of western Ecuador's Amazon region (provinces of Napo, Pastaza and Morona Santiago) and its relationship with some social and economic variables, the forests were included or not to the Socio Bosque Program. The field phase was conducted between Februarys to March 2014. We analyzed data from one hundred twenty-two surveys of these two different groups of farmers. The analysis of information included data about geographical, social, labor, economic, land use and future activities. Among other results, it is evident that most owners want to keep their wooded areas with native species, but they mention the need for more frequent training about the dynamics of local ecosystems and best ways to care for them.
\end{abstract}

Key words: ecuadorean amazon region; socio bosque programme; socio-economics; forest conservation; biodiversity loss 


\section{Introducción}

En Ecuador, los bosques nativos permanentes cubren aproximadamente 9,5 millones de hectáreas, brindan servicios ambientales y ecosistémicos constantemente. De éstas se pierden más de 60 mil hectáreas de bosques anualmente (Amores \& Jiménez, 2011).

Este hecho motivó al diseño del Programa Socio Bosque, el cual trata de dar incentivos económicos a campesinos y comunidades indígenas con un compromiso voluntario por la conservación en Ecuador. Este programa se encuentra dentro del sistema de incentivos creado por el Gobierno para la conservación de los bosques nativos remanentes y trata de obtener una distribución directa y equitativa de beneficios a nivel nacional. Está dirigido y funciona dando facilidades a los propietarios de bosques nativos, páramos y otras formaciones vegetales, el valor del incentivo depende del número de hectáreas que ingresen al programa considerando una cifra de US\$ 30,00 por hectárea por año (MAE, 2012).

Existen algunos criterios de priorización para asociar a propietarios al programa Socio Bosque como, por ejemplo, si las áreas tienen alta amenaza de deforestación, si son áreas importantes para la generación de servicios ambientales o si son áreas con altos niveles de pobreza (MAE, 2011).

El presente estudio buscó abordar algunos aspectos socio ambientales y la dinámica de la conservación de bosques nativos en los cantones Puyo, Tena y Palora, áreas donde el crecimiento demográfico es importante, afectando directa o indirectamente a sus ecosistemas locales (INEC, 2010).

\section{Materiales y métodos}

\section{Área de estudio}

La población de la Amazonía del Ecuador cuenta con indígenas de varios grupos y colonos. Los indígenas son los habitantes ancestrales de la región e incluyen ocho nacionalidades, en su mayoría ocupan vastos territorios cubiertos de bosques húmedos tropicales. Tradicionalmente han practicado la agricultura migratoria acorde con la dinámica del bosque; sin embargo, con la apertura de carreteras y la vinculación al mercado se incrementaron más sus necesidades de contar con ingresos económicos mayores (Palacios, 2010).

La presente investigación fue desarrollada en las parroquias de Ahuano, Chontapunta, Pano, Misahuallí, Puerto Napo y Tálag (cantón Tena, provincia de Napo) (Figura 1); Mera, Teniente Hugo Ortiz, Shell, Puyo, Tarqui (cantón Puyo, provincia de Pastaza) y Palora (provincia de Morona Santiago) (Figura 2).

El área de estudio fue elegida debido a que cuenta con predios de mediano tamaño y de fácil accesibilidad, además con presencia de pobladores permanentes (Granda, 2015; Clavijo, 2016). 


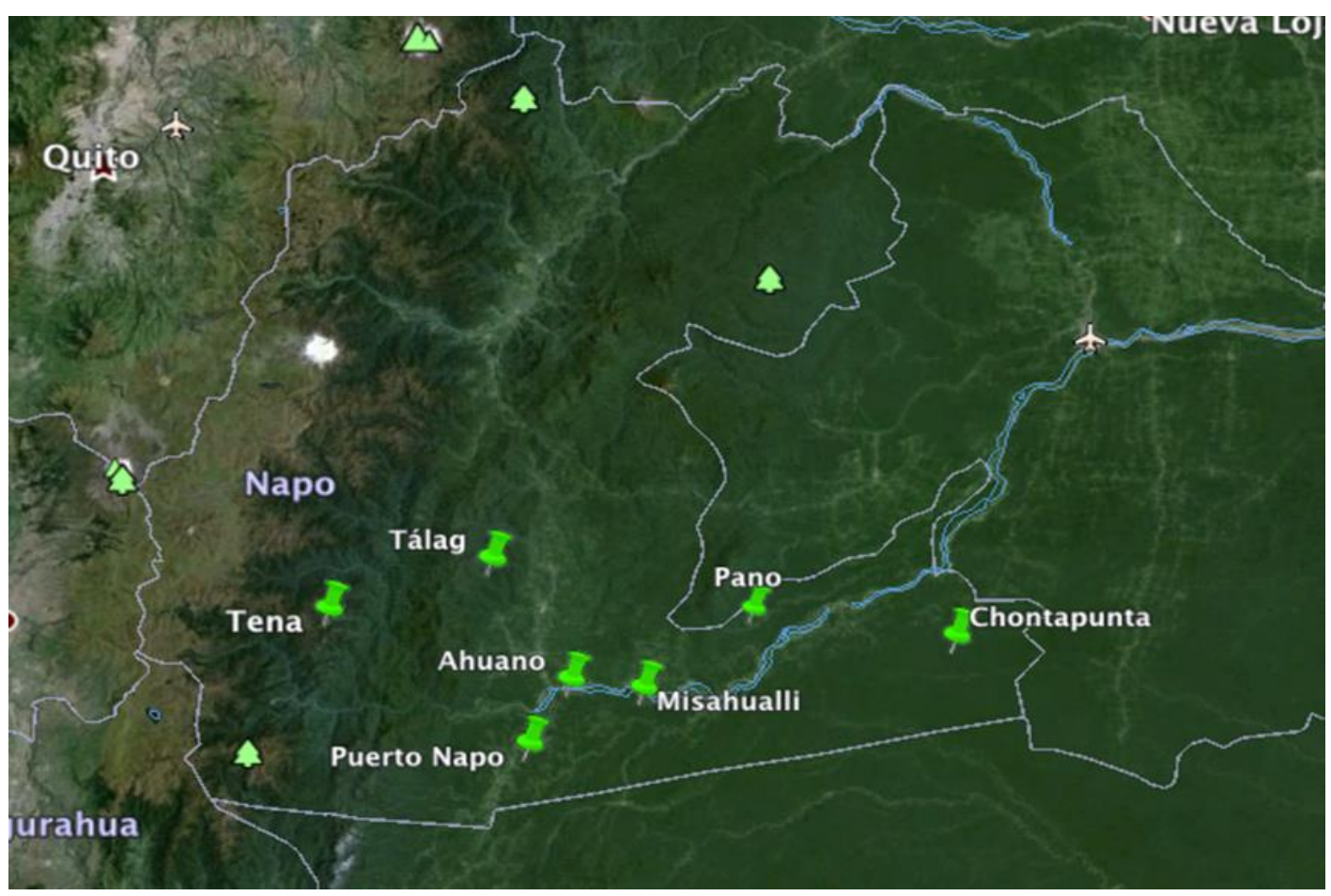

Figura 1. Parroquias de la Provincia de Napo en las que se efectuaron las encuestas.

Fuente: adaptado a partir de Google Earth, 2014.

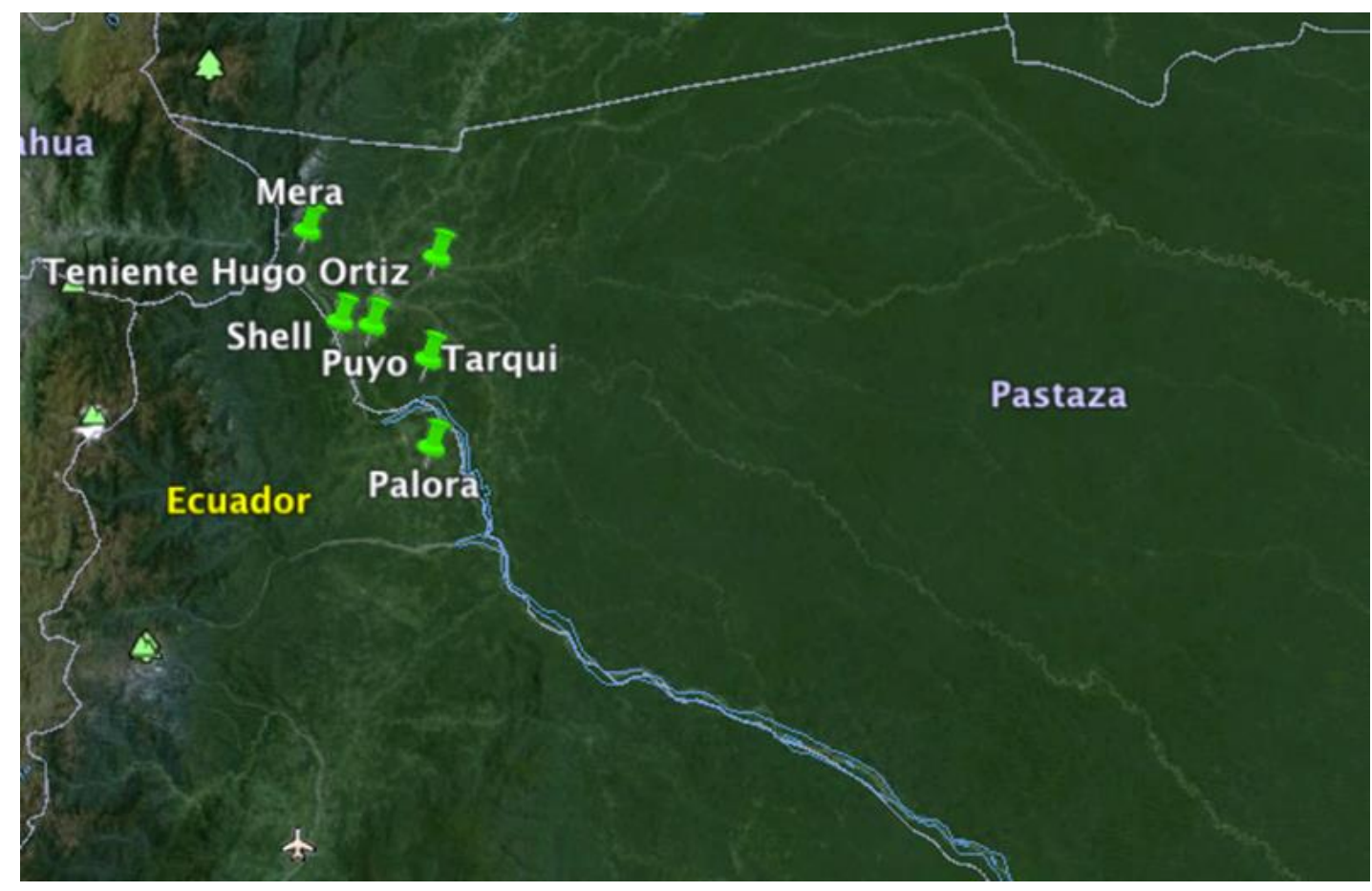

Figura 2. Parroquias de las Provincias de Pastaza y Morona Santiago en las que se aplicaron las encuestas. Fuente: adaptado a partir de Google Earth, 2014 
Entre febrero a marzo de 2014 se efectuó el trabajo de campo en 122 predios elegidos al azar (centro-occidente de las provincias de Pastaza, Morona Santiago y Napo). Se aplicó una encuesta semiabierta, misma que permitió registrar información socioambiental en cada predio, descrita con mayor detalle en los trabajos de Granda (2015) y Clavijo (2016).

En la Tabla 1 se observa el número de predios evaluados y su pertenencia a las tres provincias y a la iniciativa Socio Bosque

Tabla No. 1. Distribución de predios del estudio en cuanto a su provincia y a la pertenencia o no a Socio Bosque.

\begin{tabular}{lccc}
\hline \multicolumn{1}{c}{ Provincia } & Predios Socio Bosque & Predios No Socio Bosque & Total \\
\hline Napo & 32 & 39 & 71 \\
& 25 & 25 & 50 \\
Pastaza & 1 & 0 & 1 \\
Morona Santiago & 58 & 64 & 122 \\
Total & & & \\
\hline
\end{tabular}

\section{Aplicación y tabulación de las encuestas}

Las encuestas fueron aplicadas a través de reuniones semiformales con los propietarios, intentando siempre entablar una conversación amena y amigable.

Los grupos de preguntas aplicadas a través de la encuesta, se organizaron en una base de datos según su tipología: análisis geográfico, social, laboral, económico, uso de suelos y actividades futuras. La base de datos completa puede ser observada en los trabajos de Granda (2015) y Clavijo (2016).

\section{Análisis de la información}

Cada grupo de pregunta fue analizado por separado, primariamente con un análisis estadístico descriptivo y en los casos en los que la estructura matemática de la información lo permitía a través de un análisis multivariado (Granda, 2016) de acuerdo a lo recomendado por Fariñas (1996) y Yánez (1997a, 1997b, 2005).

\section{Resultados y Discusión}

\section{Causas que fomentan el ingreso al Programa Socio Bosque}

Al indagar por los motivos que impulsaron a los propietarios de predios a haber ingresado al programa Socio Bosque (considerando 58 propietarios que ya se encontraban en el programa, de un total de 122 encuestados) se registraron cuatro tipos de respuestas (Tabla 2).

Tabla No. 2. Causas que motivaron al ingreso de los propietarios al programa Socio Bosque 


\begin{tabular}{cccccc}
\hline Pregunta & $\begin{array}{c}\text { Conservación } \\
\text { del bosque Nativo }\end{array}$ & $\begin{array}{c}\text { Incentivo } \\
\text { Económico }\end{array}$ & $\begin{array}{c}\text { Reducción } \\
\text { de Impuestos }\end{array}$ & $\begin{array}{c}\text { Por darle algún uso no } \\
\text { extractivo a la tierra }\end{array}$ & $\begin{array}{c}\text { T } \\
\text { otal }\end{array}$ \\
\hline $\begin{array}{c}\text { ¿Por qué ingresó } \\
\text { a Socio Bosque? }\end{array}$ & 30 & 16 & 10 & 2 & 5 \\
& $(52 \%)$ & $(28 \%)$ & $(17 \%)$ & $(3 \%)$ & 8 \\
\hline
\end{tabular}

Considerando que Socio Bosque es un programa gubernamental que se creó en 2007 con el fin de apoyar y crear un incentivo para la conservación de bosques y páramos nativos en Ecuador; nótese como el 52\% de los propietarios ingresaron a SB principalmente buscando la conservación de los bosques nativos de sus propiedades. El 28\% por el Incentivo económico que brinda el programa (un monto de US $\$ 30,00$ por hectárea por año) que para ellos constituye una buena forma de ganar algo de dinero teniendo hectáreas de bosque sin alterar.

Un $17 \%$ de propietarios ingresaron a SB por la reducción de impuestos prediales que contempla la ley para socios SB. El 3\% ingresó al programa simplemente por darle algún uso a la tierra.

\section{Percepción de los beneficios brindados por los bosques nativos}

Los beneficios brindados que se perciben por la conservación del Bosque Nativo incluyen diversas situaciones ambientales y de conciencia propia (Tabla 3 ).

Tabla No. 3. Percepción sobre los beneficios que los propietarios de las fincas reciben de la conservación del Bosque Nativo.

\begin{tabular}{|c|c|c|c|c|c|}
\hline Variable & $\begin{array}{c}\text { Conservación del agua y } \\
\text { su ciclo }\end{array}$ & $\begin{array}{l}\text { Biodiversi } \\
\text { dad }\end{array}$ & $\begin{array}{l}\text { Air } \\
\text { e }\end{array}$ & $\begin{array}{l}\text { Ningu } \\
\text { na }\end{array}$ & $\begin{array}{l}\text { Tot } \\
\text { al }\end{array}$ \\
\hline $\begin{array}{l}\text { Beneficios Brindados por el } \\
\text { bosque nativo }\end{array}$ & $\begin{array}{c}43 \\
(35 \%)\end{array}$ & $\begin{array}{c}39 \\
(32 \%)\end{array}$ & $\begin{array}{c}37 \\
(30 \\
\%)\end{array}$ & $\begin{array}{c}3 \\
(2,5 \%)\end{array}$ & 122 \\
\hline
\end{tabular}

El 35\% de los propietarios perciben que el bosque nativo permite regenerar o mantener activo el ciclo del agua a nivel local, lo cual a su vez les permite proveerse de agua no contaminada cotidianamente, el $32 \%$ asocian a la conservación del bosque con el mantenimiento de la biodiversidad, el 30\% lo asocia primordialmente a la conservación con la purificación del aire, y solo un 3\% de los 122 predios estudiados no perciben ningún beneficio de la conservación de la naturaleza. Demostrando mayormente, de esta manera, que los propietarios de los predios sin haber necesariamente asistido al colegio o a la universidad poseen una alta conciencia conservacionista, ya que entienden la importancia de los recursos: agua, aire y biodiversidad y su relación estrecha con el mantenimiento del bosque.

\section{Aspectos geográficos de interés}

La mayoría de propiedades estudiadas se encuentran constituidas por predios de pequeño a mediano tamaño (4 a 110 hectáreas), con poca superficie de bosque primario (de 4 a 6 hectáreas) y relativamente poca de bosque secundario (2 a 60). 
Debido a las superficies boscosas relativamente pequeñas, la gente local tiene también pocas hectáreas dentro de Socio Bosque, por lo tanto la cantidad de dinero que reciben los dueños de los predios adscritos a esta iniciativa es relativamente baja.

También resulta importante mencionar que la mayoría de dueños de los predios no viven en el predio mismo, prefieren habitar en el centro poblado más cercano.

La presencia de pasturas para ganado oscila entre 0 a 20 hectáreas por finca. La presencia de regeneración boscosa inicial en la que predomina el arbolito Pollalesta discolor (Asteraceae) se observa en la mayoría de fincas cubriendo entre 1 a 14 hectáreas por finca; algunos estudios como los de Merino (2010) mencionan que esta regeneración inicial es una buena opción para iniciar la reforestación y uso de madera a mediano y largo plazos en la Amazonía Ecuatoriana.

\section{Aspectos sociales}

Aproximadamente la mitad de las fincas analizadas están habitadas por todos o casi todos sus propietarios e hijos; algunos dueños viven en sus fincas sin sus hijos, lo cual genera un tamaño de hogar pequeño, esto posiblemente se deba al alto índice de migración que existe en las provincias estudiadas, ya que los jóvenes generalmente salen a estudiar o a trabajar en las capitales cantonales próximas (INEC, 2010; Eche, 2014).

En general, también se observó que a mayor nivel educativo de los propietarios existe mayor interés en ingresar a Socio Bosque, en especial cuando los propietarios son jóvenes o de mediana edad.

Igualmente, es llamativo observar cómo a la gente de mayor edad (>60 años) no le interesa ingresar al programa, en general debido a que siendo o empezando a ser adultos mayores se les dificulta el cuidado de sus propiedades (INEC, 2010).

\section{Aspectos laborales}

En la mayoría de fincas adscritas al programa SB, sus propietarios no trabajan tales tierras ni efectuando agricultura ni ganadería; lo cual estaría apoyando al espíritu general del programa SB.

En cambio, en las fincas no pertenecientes a SB, casi todos los miembros de la familia trabajan la propiedad (con labores agrícolas o ganaderas) y a la vez muestran poco interés en pertenecer a SB.

\section{Análisis económico}

De las 122 fincas evaluadas (dentro y fuera de SB), tan solo unas 10 pertenecen a propietarios con buenos o muy buenos ingresos (>US\$ 1000 por familia por mes) no necesariamente derivados de SB, sino de otras actividades laborales personales (Granda, 2015). 
Finalmente, cabe destacar que casi la mitad de los propietarios de predios reciben el bono solidario principalmente las madres, que reciben US \$ 50 mensuales por parte del gobierno ecuatoriano; es importante mencionar que quienes reciben este bono tienen fincas pequeñas y no muestran interés en adscribirse a SB.

\section{Autopercepción sobre las actividades futuras}

Las cuestiones consideradas en este ámbito incluyeron: ¿Aumentará ganadería? ¿Aumentará cultivos de ciclo corto? ¿Aumentará cultivos permanentes? ¿Aumentará venta de madera? ¿Aumentará producción de animales menores? ¿Le interesa ingresar a SB? ¿Cree que el incentivo de Socio Bosque es bajo? ¿Le interesa información sobre SB? (Granda, 2015). Los resultados a estas interrogantes se pueden observar en la Tabla 4.

Tabla No. 4. Percepción de los habitantes locales sobre las Actividades Futuras que desean efectuar en sus predios

\begin{tabular}{lcccc}
\hline & \multicolumn{2}{c}{ Socio Bosque } & \multicolumn{2}{c}{ No Socio Bosque } \\
\hline \multicolumn{1}{c}{ Variables } & SI & NO & SI & NO \\
¿Aumentará Ganadería? & 2 & 56 & 4 & 60 \\
¿Aumentará Cultivos de Ciclo corto? & 4 & 54 & 7 & 57 \\
¿Aumentará Cultivos Permanentes? & 4 & 54 & 8 & 56 \\
¿Aumentará Venta de madera? & 0 & 58 & 1 & 63 \\
¿Aumentará producción animales menores? & 2 & 56 & 3 & 61 \\
¿Le interesa ingresar a Socio Bosque? & No aplica & No aplica & 39 & 25 \\
¿Cree que el incentivo de SB es bajo? & 30 & 28 & No aplica & No aplica \\
¿Le interesa más información sobre SB? & 29 & 29 & 28 & 36 \\
\hline
\end{tabular}

SB $=$ Fincas Adscritas al programa Socio Bosque $=58$.

NSB $=$ Fincas no adscritas al programa Socio Bosque $=64$.

Obsérvese como ni en las fincas SB ni en las NSB se desea aumentar la producción ganadera; este deseo mayoritario se debe a que los propietarios no encuentran un beneficio económico al tener ganado ni de carne, ni de leche, ya que en general las pasturas que se pueden producir allí poseen poco valor nutritivo para el ganado vacuno.

Con respecto a la pregunta ¿Aumentará Cultivos de Ciclo corto? se puede observar un deseo mayoritario de no querer sembrar monocultivos de ciclo corto (plátano, yuca, naranjilla, papa china y caña de azúcar) debido a que la tierra es poco fértil o la actividad agrícola tiene poco desarrollo en estas zonas; más bien las personas encuentran más rentable realizar actividades agroforestales o sembrar su chacra de manera mixta (varios productos simultáneamente en la misma superficie), lo cual técnicamente genera más beneficios que un monocultivo (Baldock, 1982; Yánez, 2006; Yánez, 2012).

En la pregunta ¿Aumentará Cultivos Permanentes? Se puede apreciar que la mayoría de propietarios tampoco desean aumentar cultivos permanentes (café y cacao); esto se explica por el 
bajo rendimiento de estas plantas en suelos amazónicos (Yánez, 2013), ya que los suelos amazónicos pierden su fertilidad pasados los 5 primeros años de cultivos intensivos (MAGAP, 2010).

Con respecto a la pregunta ¿Aumentará venta de madera? Es de suma importancia aclarar que una vez que pertenecen a SB, ya no se pueden tocar los recursos maderables incluidos en el programa. Tampoco los propietarios no adscritos a Socio Bosque desean aumentar la venta de madera extraída del bosque; esto debido a que el ecosistema forestal les brinda numerosos beneficios ambientales, entre los cuales se deben contar el agua, el aire y la biodiversidad (Yánez, 2014a, 2014b).

La negativa generalizada de no aumentar la producción de animales menores, tanto en fincas SB como en NSB, se explica debido a que la producción de gallinas, cuyes y conejos, no posee un alto rendimiento productivo, principalmente debido a que en estas zonas no existe un adecuado conocimiento con respecto al cuidado de estos animales (MAGAP, 2010).

Sobre el potencial ingreso a SB, preguntado a propietarios todavía no adscritos a este Programa, 39 desean ingresar y 25 no desean ingresar a tal iniciativa, esto posiblemente se debe a que no cumplen con el número de hectáreas requeridas, no poseen escrituras en regla de sus fincas o no están conformes con los planteamientos que propone el programa Socio Bosque (MAE, 2014).

Con respecto a la percepción sobre el incentivo brindado por SB, se puede observar que aproximadamente la mitad de los propietarios piensan que el incentivo es bueno y la otra mitad que no es adecuado. Estas opiniones posiblemente se dan debido a que existen personas que no viven de estos ingresos y poseen otros recursos monetarios que ayudan a la economía de su hogar.

En torno a la opinión de los propietarios sobre la pregunta ¿Le interesa información sobre SB? Aproximadamente a la mitad de los propietarios no SB les interesa.

\section{Sugerencias de la gente para el programa SB}

En la Tabla 5 se presentan las principales sugerencias que la gente local efectúa al Programa Socio Bosque.

Tabla No. 5. Sugerencias de la gente para la iniciativa Socio Bosque

\begin{tabular}{|c|c|c|c|c|c|}
\hline Variables & $\begin{array}{r}\text { Capaci } \\
\text { taciones }\end{array}$ & $\begin{array}{c}\text { Apoyo a } \\
\text { Reforestació } \\
n \\
\end{array}$ & $\begin{array}{r}\text { Prés } \\
\text { tamos }\end{array}$ & $\begin{array}{l}\text { Nin } \\
\text { guna }\end{array}$ & $\begin{array}{r}\text { T } \\
\text { otal }\end{array}$ \\
\hline $\begin{array}{l}\text { Sugerencias de la gente para Socio Bosque (fincas } \\
\text { adscritas más las no adscritas al programa SB) }\end{array}$ & $\begin{array}{c}45 \\
(37 \%)\end{array}$ & $\begin{array}{c}34 \\
(28 \%)\end{array}$ & $\begin{array}{c}25 \\
(20 \% \\
)\end{array}$ & $\begin{array}{l}18 \\
(15 \\
\%)\end{array}$ & $\begin{array}{r}1 \\
22\end{array}$ \\
\hline
\end{tabular}

Obsérvese como la mayor sugerencia de la gente para el programa SB (37\% de las opiniones) es que este Programa efectúe capacitaciones, que pueden ser dadas a personas 
pertenecientes como no pertenecientes a él; las mismas que pueden tratar sobre aspectos ambientales, de conservación, de producción de cultivos de manera amigable con la naturaleza.

Un $20 \%$ solicita se les ayude con préstamos para iniciar un negocio propio o para adquirir algún bien mueble o inmueble.

Otro pedido de la gente perteneciente y no perteneciente a SB es que se les ayude con actividades de reforestación; ellos demandan, por ejemplo, el inicio de programas permanentes relacionados con siembra y cuidado de árboles nativos en sus predios.

El $15 \%$ no pide nada o no tiene ninguna sugerencia ni expectativa nueva para el programa Socio Bosque.

\section{Recomendaciones}

Realizar estudios periódicos (cada 2 años al menos) que integren las variables expuestas en la presente investigación para comprender como evolucionan las actividades de conservación en la Amazonía Ecuatoriana en predios adscritos y no adscritos a SB.

Efectuar estudios biológicos de la flora y fauna en los bosques primarios y secundarios existentes en terrenos privados amazónicos, para constatar el estado actual y la dinámica de tales grupos en áreas naturales y seminaturales privadas.

Efectuar estudios sociales más amplios que aborden aspectos educativos y de edad de los propietarios para conocer cómo se involucran estas variables con la conservación de la biodiversidad en las provincias amazónicas de Ecuador.

Desarrollar un estudio económico para verificar los ingresos, egresos e ingresos netos que poseen las familias amazónicas (pertenecientes o no a SB) de manera periódica (cada 2 ó 3 años) y las actividades de conservación del bosque nativo que estas familias pudieran efectuar.

Realizar estudios sobre el uso de suelos en la Amazonía ecuatoriana, verificando por medio de sistemas de información geográfica el estado actual y la dinámica futura de la ocupación de tierras y sus relaciones con la conservación de bosques nativos.

Aumentar las conferencias y reuniones de capacitación in situ realizadas por Socio Bosque, ya que tienen una buena acogida por parte de los dueños de los predios, pudiendo a la vez aprovechar estos espacios para enfatizar los beneficios que brinda el programa.

Estas capacitaciones deben versar sobre los beneficios de la conservación de los bosques nativos, pero también sobre formas alternativas de bajo impacto ambiental en el uso de los recursos naturales locales; tales como las de ecoturismo y agroturismo (tal como lo proponen Doumet-Chilán y Yánez, 2014), turismo cultural (con casos ejemplificados por Nasimba y Cejas, 2015), manejo de productos de especies nativas (descritos en estudios como los de Yánez, 1999; Yánez, 2012, entre otros).

\section{Conclusiones}


La mayoría de personas encuestadas ingresaron a SB por la conservación del bosque nativo, seguido por el incentivo económico brindado por la iniciativa. Los propietarios de los predios estudiados se enteraron de Socio Bosque mayormente a través de la conversación con amistades, quienes les comentaron sobre los beneficios del programa.

Las personas locales asocian fuertemente la conservación del bosque nativo con la regulación de ciclos naturales, principalmente el del agua, lo cual -dicen- les permite proveerse de fuentes de agua no contaminada.

En las localidades estudiadas de las tres provincias se observa que predominan las fincas con poca superficie de bosques, debido a procesos de transformación del paisaje efectuados en décadas anteriores.

En general se observó en propietarios > 60 años de edad menor interés en pertenecer a la iniciativa SB; por el contrario los propietarios más jóvenes y de mayor nivel educativo muestran mayor predisposición a adscribirse a ella. Pudiendo mencionarse, por tanto, que la situación de conservación muestra avances positivos para el sector cuando los actores mejoran sus niveles de vida, como por ejemplo, al mejorar su educación.

Debido a los pocos ingresos que tiene la mayoría de propietarios de predios locales, ellos generalmente necesitan trabajar más fuera de la finca. Las pocas fincas que reportan mayores ingresos económicos los generan en actividades diferentes a las de Socio Bosque.

Cabe mencionar también que algunos propietarios de predios dejan que crezcan árboles naturalmente para evitar la erosión de los terrenos o porque la familia no está en capacidad de trabajar la tierra.

Algunas fincas estudiadas muestran importantes extensiones deforestadas para desarrollar agricultura y ganadería, siendo ésta seguramente la causa principal para que sus propietarios no hayan podido ingresar a la iniciativa Socio Bosque.

A pesar de ello, en la actualidad, los dueños de los predios Socio Bosque o No Socio Bosque continuamente manifiestan su deseo de no aumentar ninguna actividad productiva en sus fincas, lo cual incluye el no aumento de cultivos de ciclo corto, cultivos permanentes, ganadería, producción de animales menores y venta de madera.

A la mayoría de personas propietarias de predios que no se encuentran dentro de Socio Bosque les interesa ingresar al programa. Los dueños de predios ya pertenecientes a SB, sin embargo, creen que el incentivo económico es bajo.

Tanto los pertenecientes a SB, como los no pertenecientes, enuncian que les interesa disponer de mayor información del Programa Socio Bosque, especialmente sobre los incentivos y los beneficios brindados. 
Igualmente, las personas solicitan a Socio Bosque que ayude con capacitaciones sobre temas ambientales, agrícolas y ganaderos, también que se les ayude con más actividades de reforestación en sus predios.

\section{Bibliografía}

Amores, F., \& Jiménez, E. (2011). Evaluación de la estructura vegetal de un bosque húmedo premontano en Guasaganda. Tesis de grado en Ingeniería Agrícola. Escuela Superior Politécnica del Litoral (ESPOL). Guayaquil.

Baldock, J. W. (1982). Geología del Ecuador. Boletín del Mapa Geológico de la República del Ecuador. Dir. Geología y Minas. Ministerio de Recursos Naturales y Energéticos. Quito.

Clavijo Páez, J. C. (2016). Patrones de uso de la flora y su relación con actividades de conservación de bosques nativos en doce parroquias amazónicas de Ecuador. Trabajo de Titulación en Biología Ambiental, Universidad Internacional del Ecuador, Quito.

Doumet-Chilán, Y., \& Yánez, P. (2014). Estrategias para desarrollar el agroturismo en la represa Sixto Durán Ballén, Manabí, Ecuador. Espamciencia, 5(1), 7-15.

Eche, D. (2014). El trabajo decente y el abandono de la agricultura a pequeña escala en Ecuador. Qualitas, 8, 24-54.

Fariñas, M.R. (1996). Análisis de la Vegetación y de sus relaciones con el ambiente mediante Métodos de Ordenamiento. Trabajo de Ascenso. Universidad de Los Andes. Mérida, Venezuela.

Granda Muñoz, M. J. (2015). Análisis Socio-Ambiental en doce parroquias amazónicas de Ecuador y su relación con actividades de conservación de bosques nativos. Trabajo de Titulación en Biología Ambiental, Universidad Internacional del Ecuador, Quito.

Google Earth. (2014). Imágenes de la Región Amazónica Ecuatoriana. Revisadas entre mayo a agosto de 2014.

INEC: Instituto Nacional de Estadística y Censos. (2010). Resultados del Censo 2010 de población $y$ vivienda en el Ecuador para Pastaza y Napo. Ecuador. Recuperado de http://www.ecuadorencifras.gob.ec/censo-de-poblacion-y-vivienda/; Revisado el 5 de noviembre de 2014.

MAE. Ministerio del Ambiente de Ecuador. (2011). Estimación de la Tasa de Deforestación del Ecuador continental. Quito: MAE.

MAE: Ministerio del Ambiente de Ecuador. (2012). Informe técnico del mapa histórico de deforestación para los períodos 1990, 2000 y 2008. Ministerio del Ambiente. Informe no publicado. Quito. Recuperado de http://www.ambiente.gob.ec/mae-iniciara-proceso- 
administrativo-por-deforestacion-de-17-hectareas-de-bosque-primario-en-manabi/; revisado el 4 de mayo del 2014.

MAE. Ministerio de Ambiente del Ecuador. (2014). Experiencia en Compensación por Servicios Ambientales en América Latina (PSA o REDD+). Quito.

MAGAP. Ministerio de Agricultura, Ganadería, Acuacultura y Pesca. (2010). Proyecto Plan Tierras. Quito - Ecuador.

Merino, J. 2010. Estudio económico de dos formas de aprovechamiento forestal del Pigüe (Pollalesta discolor) en el cantón Mera, provincia de Pastaza. Tesis de grado para la obtención del título ingeniero forestal. Riobamba. Ecuador.

Nasimba, C. y Cejas, M. (2015). Diseño de productos turísticos y sus facilidades. Qualitas, 10, 2239.

Palacios, W. (2010). Situación de las comunidades productoras forestales de la Amazonía Ecuatoriana: obstáculos y oportunidades para comercializar madera legal. TRAFFIC América del Sur, VERIFOR. Quito: Options For Forest Verification.

Yánez, A. P. (1997a). Seminario Bibliográfico "Distribución de especies vegetales en ecosistemas naturales desde la óptica del Continuum: Evolución de la Idea y algunos Métodos para su Caracterización”. CIELAT, Universidad de Los Andes, Mérida, Venezuela, 44pp.

Yánez, A. P. (1997b). Análisis de la distribución de especies vegetales a lo largo de un gradiente altitudinal Páramo-Selva Nublada del Parque Nacional Sierra Nevada, Venezuela. Tesis de Maestría en Ecología Tropical. Universidad de Los Andes. Mérida, Venezuela.

Yánez, P. (1999). Distribución geográfica y aspectos etnobotánicos de tres especies del género Pourouma ("uva de monte"), Cecropiaceae, en la región amazónica de Ecuador. Rev. Forest. Venez, 43(1), 103-109.

Yánez, P. (2005). Biometría y Bioestadística fundamentales. Analizando la estructura numérica de la información en proyectos ecológicos. Quito.

Yánez, P. (2006). Plan de uso y manejo de la guaviduca (Piper carpunya Ruiz \& Pav., Piperaceae) para la comunidad de Chiriboga y áreas adyacentes, Pichincha-Ecuador. Programa de Manejo de la Biodiversidad y Biocomercio (PMBB)-EcoCiencia. Informe final presentado al Programa de Facilitación del Biocomercio/UNCTAD. Quito.

Yánez, P. (2012). Consideraciones para el diseño y aplicación de planes de manejo de especies vegetales silvestres no maderables de interés comercial. Qualitas, 4, 31-40.

Yánez, P. (2013). La pérdida de los bosques tropicales: algunos de sus efectos sobre la estabilidad de nuestro Planeta. Qualitas, 6, 74-78. 
Yánez, P. (2014a). Ecología y biodiversidad: un enfoque desde el neotrópico. Quito: UNIBE/UIDE. 172pp.

Yánez, P. (2014b). Un vistazo a la conservación Biológica del Ecuador. Qualitas, 7, 80-84. 